\title{
THERMOMETRIC LAG OF AIRCRAFT THERMOMETERS, THERMOGRAPHS, AND BAROGRAPHS
}

\author{
By H. B. Henrickson
}

\section{ABSTRACT}

A constant which will be called the time lag has been determined experimentally for thermographs, barographs, and of the temperature element of a Fergusson meteorograph. When the instruments are exposed in the same medium and to the same ventilation this constant can be used to evalute their thermometric lag. For the purpose of comparison this constant was also measured for a number of laboratory thermometers. The tests on the thermometers and thermographs were made in an air stream of $17 \mathrm{~m} . \mathrm{p} . \mathrm{h}$. and those on the barographs in still air and in an air stream of $10 \mathrm{~m}$. p. h. Results for the thermometers show that the liquid-filled type has the greatest time lag and therefore under similar conditions of exposure the greatest thermometric lag, followed in order by the bimetallic strip type and the electric resistance thermometers. All of the barographs, even the metal-cased types, have a far greater time lag than the thermometers, but the lag is reduced considerably by moderate ventilation. The lag of the thermometers ranged from 3 to 72 seconds, whereas that of the barographs in still air ranged from 22 to 64 minutes. The lag of the temperature element of the meteorograph was found to be 3 minutes in still air and 11 seconds in an air stream of 17 miles per hour.

\section{CONTENTS}

I. Introduction

II. Relation of time lag and thermometric lag

III. Method of determining lag and experimental procedure

IY. Results of tests on thermometers and thermographs_....... 700

V. Results of tests on barographs... 707

VI. Summary

1. Thermometers, thermographs, and meteorograph

2. Barographs .......... 708

\section{INTRODUCTION}

The thermometric lag of aircraft thermometers used to measure air temperatures may introduce considerable error when the instruments are used under conditions where the air temperature is changing. Such conditions exist, for example, during the climb of an aircraft or a sounding balloon, and here the lag of the air thermometer must be considered if accurate measurements are desired.

Determinations have been made of a constant, which is called the time lag, for a representative group of indicating and recording instruments which were selected from those available from time to time during the past five years. A number of the temperature elements, including experimental types, of the electric resistance thermometers which were constructed for the National Advisory Committee for Aeronautics and the Bureau of Aeronautics of the United States Navy have also been tested. The cooperation of these organizations, 
of the Weather Bureau, and of the various manufacturers who submitted instruments for the investigation is acknowledged.

Owing to their value to the designer of instruments and perhaps to others, data have also been obtained on a number of thermometers of types not ordinarily used in aircraft.

In addition, measurements of this constant have been made on representative types of barographs. The data obtained are of particular value in computing the thermal lag of barographs during their test in the laboratory for the effect of temperature, and thus furnishing in a measure the minimum time required for the instrument to come to any chosen degree of temperature equilibrium in the temperature chamber. Further, when no provision is made for the measurement of the instrument temperature either in flight tests or in high altitude flights, the approximate temperature of the instrument may be computed from a knowledge of the time lag, together with data on the ventilation of the instrument during flight and the air temperature at the ground level. This temperature is useful in estimating or determining the error introduced by the variation of the temperature of the barograph.

\section{RELATION OF TIME LAG AND THERIMOMETRIC LAG}

$\Lambda$ detailed discussion of the question of thermometric lag is given in a paper by Harper. ${ }^{1}$ The method, apparatus, and procedure are described in a previous paper. ${ }^{2}$ With some modifications this is repeated here for ready reference.

In general, a problem of thermometric lag can be treated with sufficient accuracy by assuming that Newton's law of cooling applies; that is, that the rate of change in the temperature of the thermometer is proportional to the difference between this temperature and that of the surrounding medium. If $T$ be the temperature of the thermometer or instrument, $t$ the time, and $U$ the temperature of the medium Newton's law is expressed by the equation-

$$
\frac{d T}{d t}=\frac{1}{\lambda}(U-T)
$$

where $\lambda$ is a constant which will be called the time lag, since it has the dimensions of time.

The equation is written so as to make $\lambda$ a positive constant, for if $\lambda$ is positive, and $U$ is higher than $T, d T / d t$ will also be positive. The constant of proportionality is written in the form $1 / \lambda$ for convenience, because when so written $\lambda$ can bo interpreted in a simplo and useful manner.

Two cases differing as to the temperature, $U$, of the bath or surrounding medium are of interest here. In the first case the temperature has a constant value $U_{0}$, that is-

$$
U=U_{0}
$$

In the second, the temperature varies uniformly with time at a rate $r$, that is-

$$
U=U_{0}+r t
$$

1 Thermometric Lag, B. S. Bull., 8, p. 659-714; 1912. (S., 185.)

2 II. B. IIenrickson and W. (.. Brombacher, Lag of Thermometers and Thermographs for Aircraft, Mo. Weather Rev., 55, p. 72-73; 1927. 
Integrating equation (1) on the basis of the assumption expressed in equation (2) gives the relation-

$$
\log _{e} \frac{T_{0}-U_{0}}{T-U_{0}}=\frac{t}{\lambda}
$$

In this equation $U_{0}$ is the temperature of the bath or medium in which the thermometer or instrument is placed, $T_{0}$ the initial temperature of the thermometer at time $t=0$, and $T$ the temperature of the thermometer, at time $t$.

If on the other hand, the temperature of the bath is changing in the manner defined in equation (3), the integration of equation ( 1 ' gives the expression-

$$
T-U=-r \lambda+\left(T_{0}-U_{0}+r \lambda\right) e^{-\overline{\bar{\lambda}} t}
$$

When $t$, the time in which the instrument has been in the bath, is large compared with $\lambda$, the time lag, the last term of the righthand member of equation (5) becomes negligible. This condition is the most common one. Equation (5) then reduces to-

$$
T-U=-r \lambda
$$

In equations (5) and (6), $U_{0}$ and $T_{0}$ are the initial temperatures, at time $t=0$, of the bath and the thermometer, respectively; $U$ and $T$ are the corresponding temperatures at time $t ; r$ is the rate of change of temperature of the bath; and $e$ is the base of natural logarithms (2.72).

It follows from equation (4), that if a thermometer be plunged into a bath maintained at a constant temperature (the thermometer being initially at a different temperature), $\lambda$ is the number of seconds in which the difference between the thermometer reading and the bath temperature is reduced to $e^{-1}$ times its initial value.

The time required to reduce the difference in temperature. between the thermometer and the bath to any fractional amount of the original difference can be easily computed from equation (4). The computation shows that the difference in temperature reduces to $0.1,0.02$, and 0.01 of its original value in a time of $2.3 \lambda, 3.9 \lambda$, and $4.6 \lambda$, respectively. For example, if a thermometer having a time lag of 10 seconds under the conditions of use is initially at a temperature of $+20^{\circ} \mathrm{C}$. and is then placed in a bath at $-30^{\circ} \mathrm{C}$., it will indicate $-25^{\circ} \mathrm{C}$. in 23 seconds, $-29^{\circ} \mathrm{C}$. in 39 seconds, and $-29.5^{\circ} \mathrm{C}$. in 46 seconds.

As a further example which is of some practical interest in testing instruments, assume an instrument to have a lag of 20 minutes in still air, and to be initially at a temperature of $+20^{\circ} \mathrm{C}$. If it is put into a test chamber held constant at a temperature of $-30^{\circ}$ C., the air in the chamber being at rest, the instrument temperature will drop to $-25^{\circ} \mathrm{C}$. in $2.3 \times 20$ or 46 minutes, and to $-29^{\circ} \mathrm{C}$. in 78 minutes, or 1.3 hours.

For the conditions under which equation (6) applies, the following interpretation obtains:

If a thermometer has been immersed for a long time in a bath whose temperature is rising at uniform rate, $\lambda$ is the number of seconds between the time when the bath attains any given tempera$4754^{\circ}-30-13$ 
ture and the time when the thermometer indicates this temperature. In other words, it is the number of seconds the thermometer "lags" behind such a temperature.

As is evident from equation (6), the difference between the temperature of the bath and the thermometer, is the product of the time lag and the rate at which the temperature of the medium or bath is changing.

The thermometric lag is of especial importance in the measurement of air temperature during the climb of an aircraft. In the performance testing of aircraft a maximum rate of climb is usually maintained, and, therefore, the temperature of the free air in the vicinity of the aircraft is decreasing. Although the rate of decrease of temperature is by no means constant owing to the falling off in the rate of climb with altitude and the possible existence of inversions in the air temperature gradient, the order of magnitude of the difference in temperature between the thermometer and the air can be evaluated by equation (6). It may be assumed that the time lag of a thermometer increases as the density of the air decreases or as the altitude increases, which in some measure offsets the effect due to the falling off in rateof-climb with altitude. With these reservations in mind, assume that an airplane climbs at a rate of 3,000 feet per minute in an atmosphere in which the average conditions of free air temperature exist. The value of $r$ is then approximately $0.1^{\circ} \mathrm{C}$. per second. Further, assume that the time lag, $\lambda$, of the thermometer when exposed to the free air moving at the air speed of the airplane is 10 seconds. Inserting these values in equation (6) it is seen that $(T-U)$ equals $1^{\circ} \mathrm{C}$., which means that the indication of the thermometer lags $1^{\circ} \mathrm{C}$. behind the free air temperature. If the time lag is assumed to be 30 seconds, the indication lags $3^{\circ} \mathrm{C}$.

The indications of instruments are affected by changes in temperature. This fact is of particular importance in the case of barographs used in the measurement of air pressure at high altitudes. The approximate temperature of the instrument must be known in order to determine the pressure accurately. Since the time lag, $\lambda$, is relatively large for barographs, equation (5) applies, and not the simpler equation (6). A computation shows that if $T_{0}$ is equal to $U_{0}$, the temperature differences given by equations (5) and (6) are the same within 2 per cent when $t$ exceeds $4 \lambda$. As an example, assume that a barograph has a time lag of 20 minutes under the conditions of use and that the temperature of the surrounding air decreases by $3^{\circ} \mathrm{C}$. per minute. The temperature differences on these assumptions determined by both equations (5) and (6) are given in Table 1.

TABLE 1

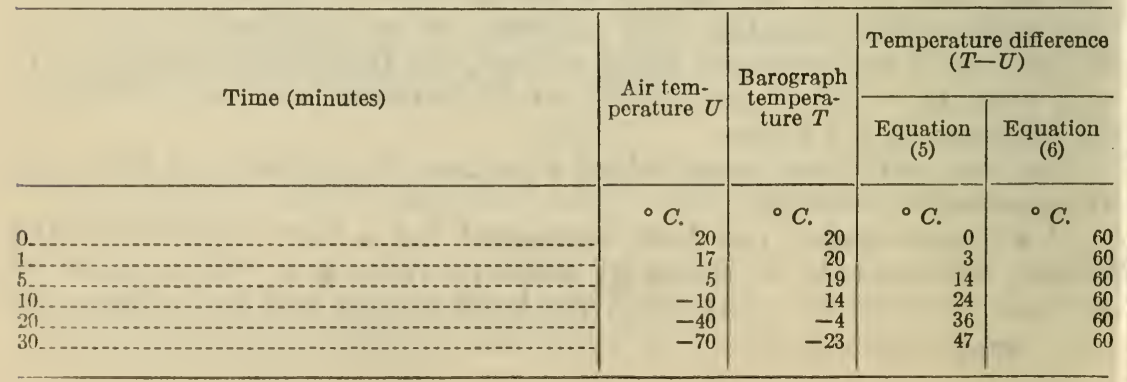


It is recognized that the temperatures to which a barograph may be subjected during flight do not follow the simple assumption of a uniform rate of decrease. In fact, actual conditions are such that a substantially uniform decrease of temperature is experienced during the first part of a climb, followed by a period during which the temperature of the surrounding air is practically constant. Such cases can be dealt with by application of both formulas (4) and (5).

\section{METHOD OF DETERMINING LAG AND EXPERIMENTAL PROCEDURE}

Equation (4) was adopted as the basis for the method of determining the time lag $\lambda$, of the thermometers and barographs. For this purpose, the equation is written in the form

$$
\lambda=\frac{t}{\log _{e} \frac{T_{0}-U_{0}}{T-U_{0}}}
$$

in which the notation is the same as that previously given.

The temperature elements of the thermometers under test were brought to a temperature $T_{0}=0^{\circ} \mathrm{C}$. by packing them in ice, and the barographs and meteorograph to $T_{0}=-20^{\circ} \mathrm{C}$. by placing them in a cold chamber. Thin sheet metal containers, just large enough to hold the various thermometer bulbs, were used to keep the bulbs dry while packed in cracked ice. Each instrument was then suddenly immersed in the air stream of a wind tunnel at room temperature $U_{0}$, which was approximately $+25^{\circ} \mathrm{C}$. Observations were made of the changing readings of the thermometers at known intervals of time. The temperature of the air in the wind tunnel, $U_{0}$, was also noted and was found to be constant during the short period of time required to obtain the wind-tunnel observations, usually about one minute. In the case of the barograph tests this time was decidedly longer, but the change in room temperature was always found to be negligible. Any inaccuracies in this connection would result in marked deviations from a straight line of the points at the right ends of such curves as are plotted in Figures 1 or 2 .

The wind tunnel consisted of a cardboard tube 10 inches in diameter built around two electric fans, one placed at each end of the tunnel. An opening was cut in the middle of the tube and a Pitot-Venturi nozzle of known calibration was inserted in order to measure the air speed. The pressures developed by the nozzle were observed on a water manometer. On 110 volts the fans developed an air speed of 10 miles per hour; and on 220 volts, an air speed of 17 miles per hour.

The bulbs of all indicating instruments were placed in the wind tunnel so that the long axis of each instrument or element was at right angles to the axis of the wind tunnel. The principal planes of the temperature elements of the thermographs were parallel to the axis of the wind tunnel during the tests. In the case of the Fergusson meteorograph, the air channel of the instrument was placed at an angle of approximately $45^{\circ}$ with the axis of the wind tunnel. All barographs were tested with their covers in place and with their long: axes parallel to that of the wind tunnel. 


\section{RESULTS OF TESTS ON THERMOMETERS AND THER- MOGRAPHS}

The time $t$, plotted against the ratio

$$
\frac{T_{0}-U_{0}}{T-U_{0}}
$$

is shown in Figures 1 and 2 for representative instruments. The ratio is plotted on a logarithmic scale using common logarithms. The slope of the line is the time lag, $\lambda$, provided that the abscissas are converted to logarithms to the base $e$. A brief description of

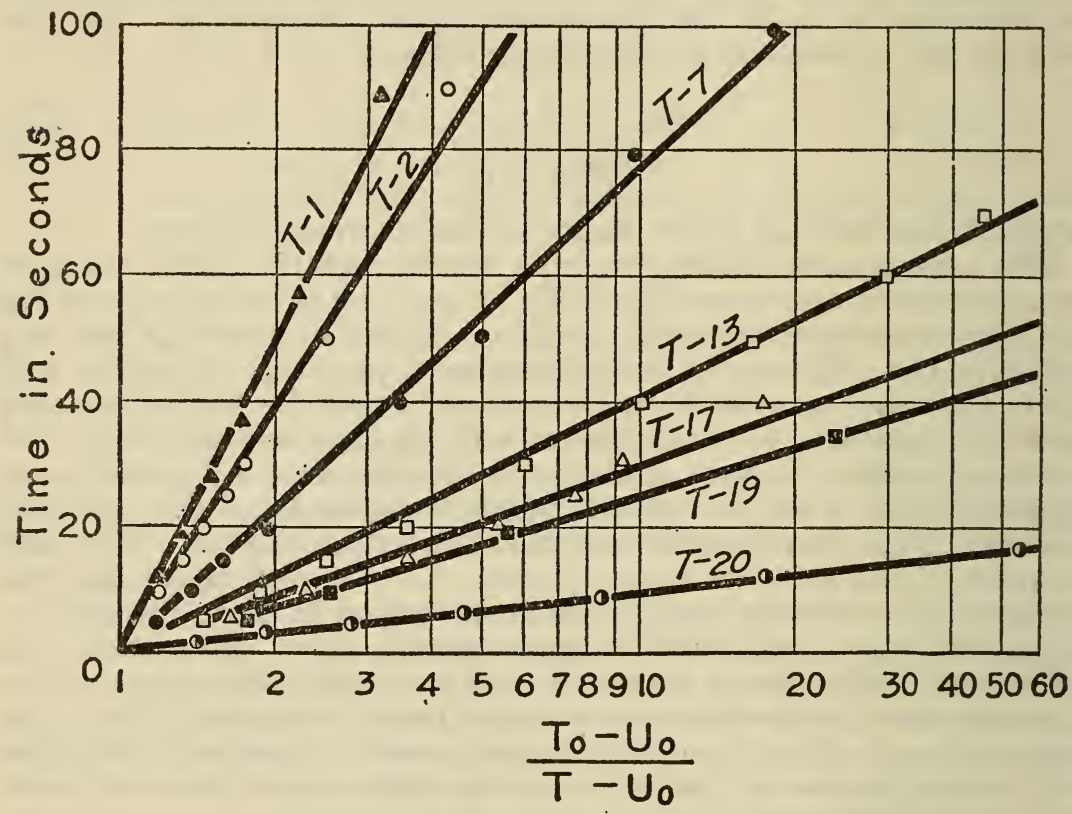

FigURE 1.-Data on thermometers and thermographs in an air stream of 17 miles per hour

The time in seconds required for the instrument to attain a temperature of $\mathrm{T}^{\circ} \mathrm{C}$. is shown as a function of the quantity

$$
\frac{T_{0}-U_{0}}{T-U_{0}}
$$

where $T_{0}$ is the initial temperature of the instrument and $U_{0}$ the temperature of the medium in ${ }^{\circ} \mathrm{C}$.

the instruments, including values determined for the lag, is given below. Sketches of representative thermometer bulbs are given in Figures 3 and 7 , and their lags in an air stream of 17 miles per hour are given in tabular form in the summary, the bulbs being arranged in the order of decreasing lag values.

Data given by Harper for a "chemical" mercurial thermometer cover a range from 0 to about 24 miles per hour. Extension to flying speeds involves a very considerable extrapolation, but by plotting $1 / \lambda$ against the logarithm of the speed the curve can be approximately rectified. Extrapolation of this rectified curve indicates that at flying speeds the lags of all the thermometers described 
below would be reduced to approximately one-half of those determined at the air speed of 17 miles per hour. The data for two air speeds given for instruments $\mathrm{T}-15$ and $\mathrm{T}-22$ in the following, also indicate that at flying speeds the lag would be about one-half of that determined at 17 miles per hour.

The thermometers tested may be conveniently divided into the following types: Liquid-filled, vapor-pressure, liquid-in-glass, bimetal, and electric-resistance. Of these the liquid-filled, vapor-pressure and electric-resistance types may be used for distant indication.

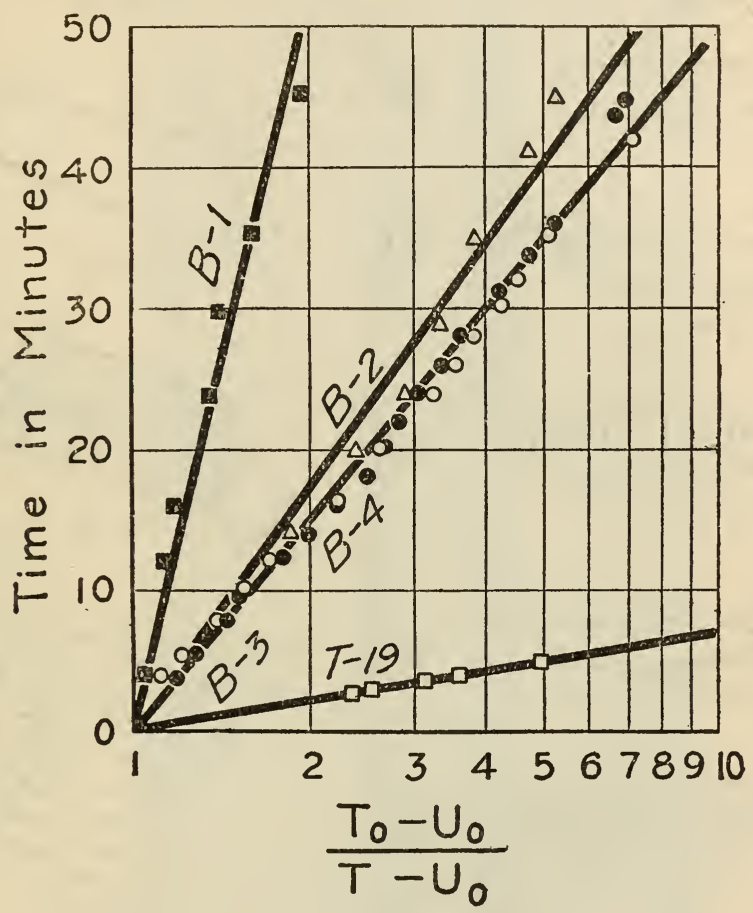

FigdRE 2.-Data on barographs and a meteorograph in still air

The time in minutes required for the instrument to attain a temperature of $\mathrm{T}^{\circ} \mathrm{C}$. is shown as a function of the quantity

$$
\frac{T_{0}-U_{0}}{T-U_{0}}
$$

where $T_{0}$ is the initial temperature of the instrument and $U_{0}$ the temperature of the medium in ${ }^{\circ} \mathrm{C}$.

It is obvious that the time lag of an instrument of any given type depends largely upon the design of that particular instrument. Thus for the liquid-in-glass instruments, the time lags given below range from 17 to 57 seconds in an air stream of 17 miles per hour. The design in turn must meet the service requirements, the effect of which is illustrated by the difference in the time lags of the liquid-in-glass strut and laboratory thermometers. The lags range from 32 to 57 seconds for the strut type, and from 17 to 28 seconds for the laboratory type. However, allowing for the differences in time lag of individual designs of a given type of thermometer, each type is inherently limited in the lowest value which may be obtained. Thus 

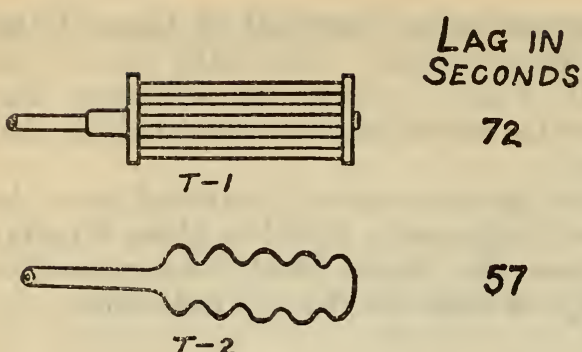

72

57

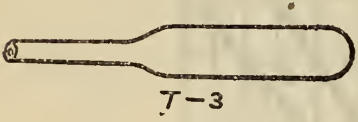

52

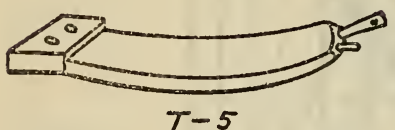

46

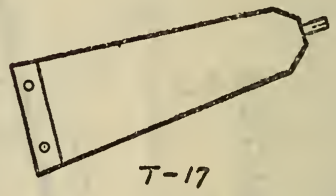

LAG IN SECONDS

72

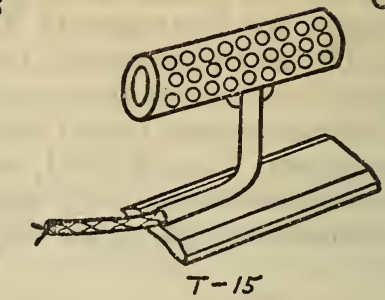

17

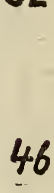

13

39

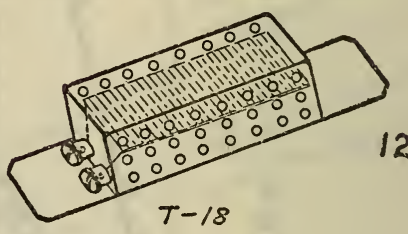

B
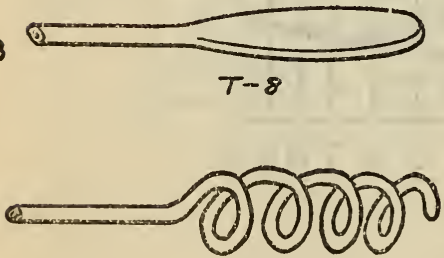

$T-3$
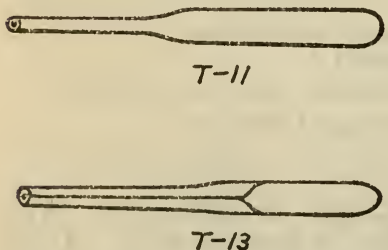

30

32

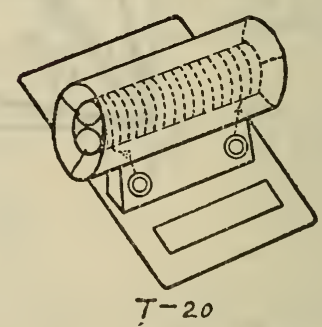

28

18

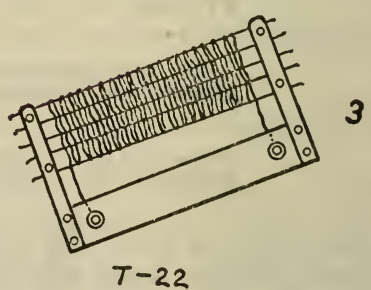

Figure 7.-Sketches of various types of temperature elements for electricresistance thermometers and of a bimetal element

$\mathrm{T}-15$, electric resistance element, three layers No. 36 nickel wire; T-17, bi-metal strip element, 0.023 inch thick; $T-18$, electric resistance element, two layers No. 36 nickel wire; T-20, electric resistance element, one layer No. 40 nickel wire; T-22, wire weave resistance element, one layer No. 40 nickel wire. 
B. S. Journal of Research, RP222

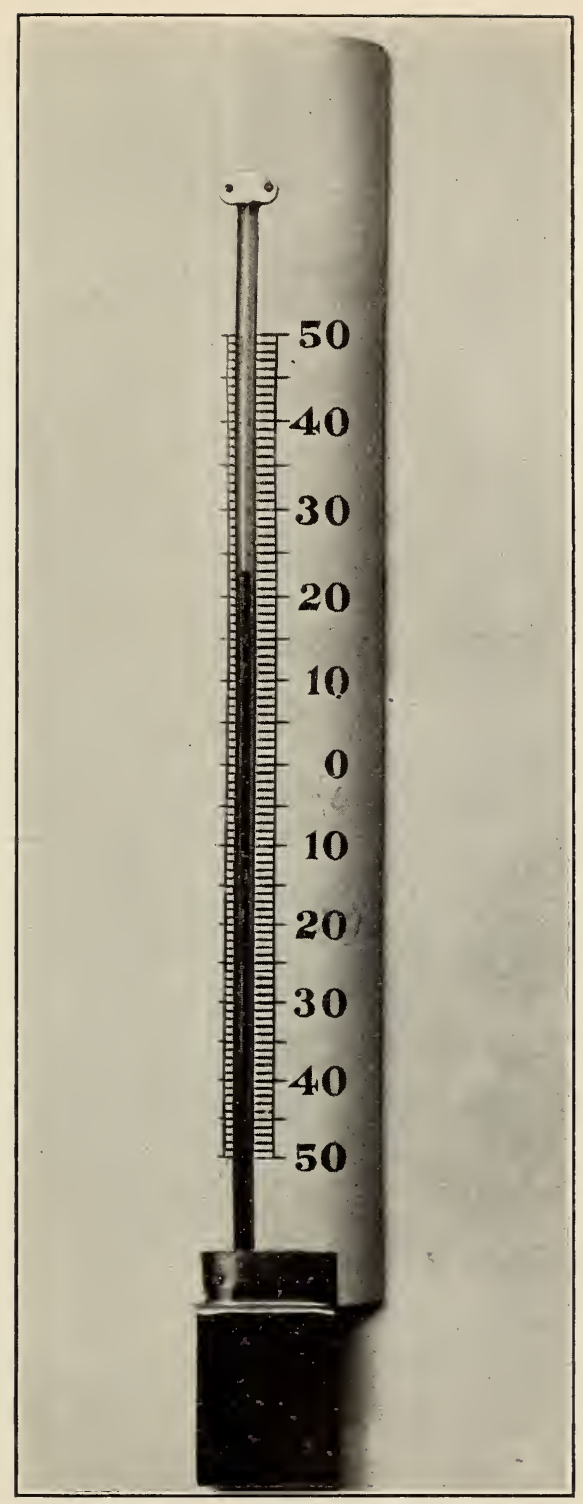

FIGURE 4.-Airplane strut thermometer for measuring free air temperature 


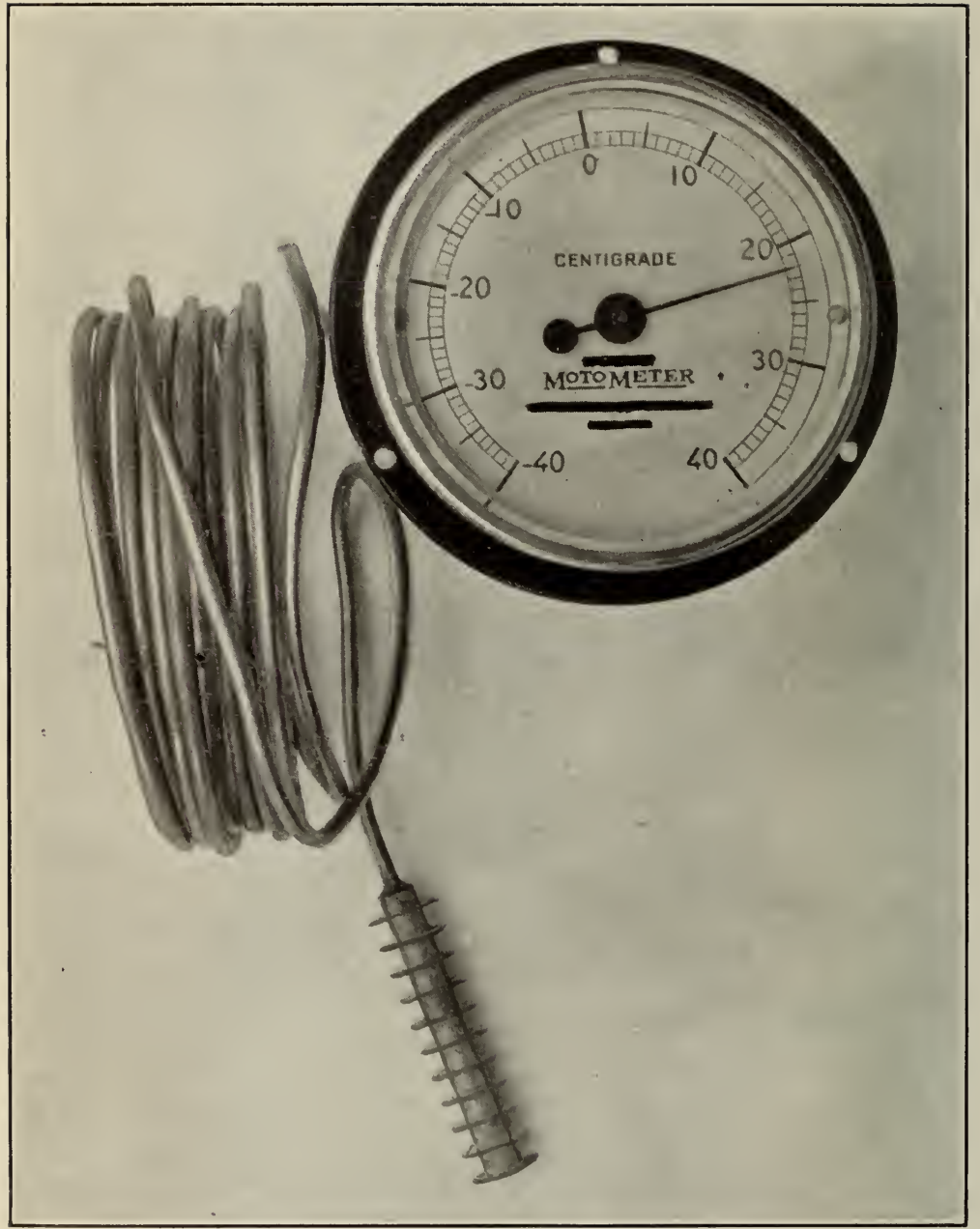

Figure 5.-Liquid-filled, distant indicating aircraft thermometer 
B. S. Journal of Research, RP222

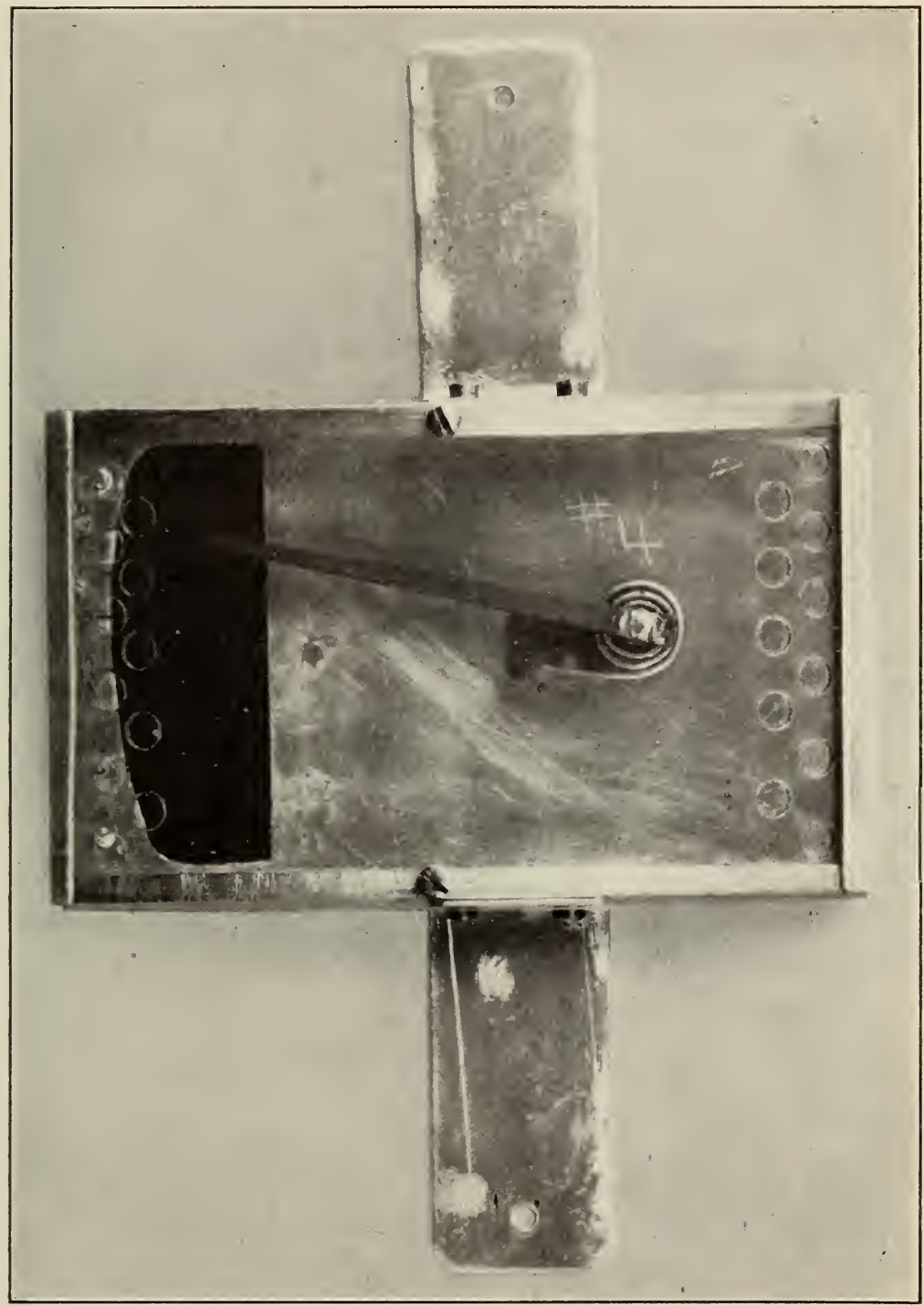

FiguRe 6.-Minimum temperature recording strut thermometer 
B. S. Journal of Research, RP222

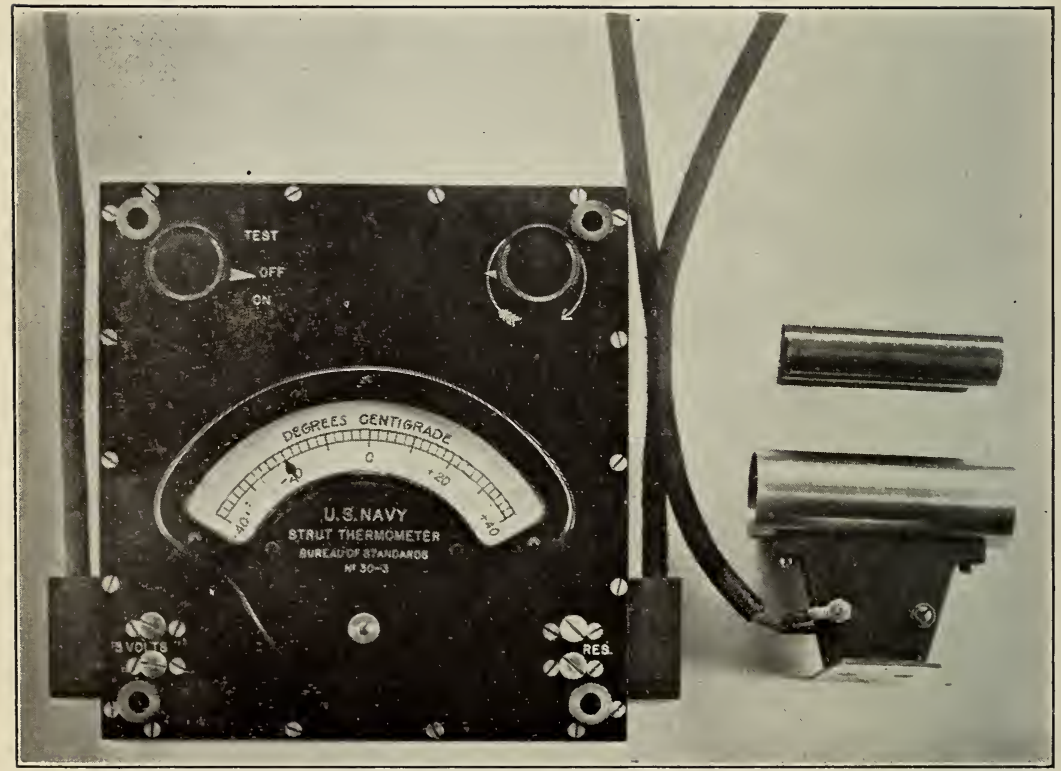

FIGURE 8.-Electric resistance thermometer for measuring free air temperature The temperature element is shown to the right, and above it the resistance coil. 
the data show that a thermograph with a bimetal element can be made with a lower lag than one with a liquid-filled Bourdon tube. In general, the time lag of properly designed liquid-filled elements of all types usually exceeds that of bimetal elements which in turn exceeds that of comparable resistance elements. It is realized that other factors enter into the choice of a type to be used so that the final decision is usually a compromise.

The time lags given in the following list are those for exposure in an air stream of 17 miles per hour unless otherwise noted.

T-1. Thermograph with liquid-in-metal-tubes bulb.-This instrument was originally designed to record free air temperature. The bulb was to be mounted on the strut and the recorder in the cockpit. The instrument also contained an element for recording the air temperature in the cockpit, the lag of which is reported under T-5. The tubular bulb, consisting of five metal tubes arranged in the form of a griciron, was unusually large. Each tube measured 7 inches in length by 0.19 inch in external diameter. In addition to having a considerable mass of liquid, the element also had a considerable mass of metal. The bulb is represented in sketch T-1 of Figure 3. Time lig, $\lambda, 72$ seconds.

T-2. Strut thermometer, liquid-in-glass bulb.-This is a columntype instrument such as is shown in Figure 4 and is designed for use as an airplane strut thermometer. The corrugated cylindrical bulb of this instrument was unusually large compared with the ordinary chemical thermometer, and contained a considerable mass of glass and liquid. It measured 1.75 inches in length by 0.43 inch in diameter. It was protected by a metal sun shield which was perforated at the leading and trailing ends so as to permit air-flow around the bulb. The over-all dimensions of the entire thermometer were 26.5 inches in length, by 3.75 inches in width, by 1 inch in thickness. The bulb itself was like that shown in sketch T-2 of Figure 3. Lag, 57 seconds.

T-3. Strut thermometer, liquid-in-glass bulb.-This instrument was of the same make as T-2, but of slightly different bulb design, as may be seen by comparing sketches T-2 and $\mathrm{T}-3$ of Figure 3 . The bulb was 2 inches long, and of circular cross section measuring 0.31 inch in diameter. This bulb also contained a considerable mass of liquid. Lag, 52 seconds.

T-4. Radiator thermometer, vapor-pressure type.-This type of instrument is commonly used to measure the temperature of the cooling water or oil of an airplane motor. The instrument had a metal bulb 3.5 inches long, with a circular cross section measuring 0.44 inch in diameter. Lag, 48 seconds.

T-5. Thermograph, Bourdon tube liquid-filled.-This unit was the part of the instrument described under T-1 which measures the air temperature in the cockpit. The Bourdon tube was 2.5 inches long, 1.25 inches wide, and 0.12 inch thick. A sketch of the element is shown in T-5 of Figure 3. Lag, 46 seconds.

T-6. Strut thermometer, distant reading, liquid-filled.-This instrument had a liquid-filled bulb with 11 radiation disks placed along its length. The bulb was 3.1 inches long, and had a circular cross section which measured 0.4 inch in diameter. The outside diameter of the radiation disks was 0.75 inch. The bulb is shown in sketch 
T-6 of Figure 3. A photograph of the entire instrument is shown in Figure 5. Lag, 39 seconds.

T-7. Thermograph, Bourdon tube, liquid-filled.-The Bourdon tube was 2.25 inches long, 0.62 inch wide, and 0.06 inch thick. The temperature element was similar to that shown in sketch T-5 of Figure 3. The over-all dimensions of the instrument were 6.5 inches in length, by 8 inches in height, by 3.75 inches in width. Lag, 33 seconds.

T-8. Strut thermometer, liquid-in-glass.-This instrument was somewhat similar to T-3 described above, including also the cover for protecting the bulb from the sun. The instrument differed from T-3 in that the bulb was much better streamlined and had less mass. Instead of a circular cross section, the bulb had a very flat elliptical cross section with the narrow edge heading into the air stream. The length of the bulb was 3.25 inches, the width 0.50 inch, and the thickness 0.19 inch. A sketch of it is shown in T-8 of Figure 3. Front and profile views of the instrument are shown in Figure 4. Lag, 32 seconds.

T-9. Strut thermometer, liquid-in-metal helical bulb.-This instrument was of the same general type as T-6 described above, except that the bulb in this case was made in the form of a long tubular helix of approximately five turns. The over-all length of the coiled bulb was 5.5 inches, and the diameter of the tube was 0.44 inch. The bulb of this instrument is shown in the sketch T-9 of Figure 3. Lag, 30 seconds.

T-10. Minimum-temperature-recording strut thermometer.-This instrument was designed primarily for recording the minimum temperature of the free air at the ceiling of high altitude flights. A bimetallic strip of brass and invar-steel, with a thickness of 0.030 inch and a width of 0.25 inch, coiled into a flat spiral of two and onehalf turns, was securely attached to an aluminum base plate which could be taped to the strut. A rectangular piece of celluloid, bent over a streamlined form, served as a cover that had the advantage of being transparent. The leading and trailing edges of this cover were perforated in order to allow the air stream to ventilate effectively the temperature element. The weight of the entire instrument was 2 ounces. A photograph of the device is shown in Figure 6. Lag, 29 seconds.

The same bimetal coil with pointer attached was removed from its casing, and mounted on a thin flat piece of bakelite 0.06 inch thick, by 2.5 inches wide, by 4.25 inches long. Without covering of any kind, the coil was placed in the wind tunnel in such a position that the air stream could readily flow through its convolutions. The lag, when determined in this manner in an air stream of 17 miles per hour, was found to be reduced to 10 seconds.

T-11. Laboratory thermometer, liquid-in-glass type.-The bulb of this thermometer was cylindrical in shape, 0.75 inch in length by 0.22 inch in diameter. The over-all length was 12 inches. The liquid was believed to be colored pentane. This bulb is shown in sketch T-11 of Figure 3. Lag, 28 seconds.

T-12. Laboratory thermometer, liquid-in-glass type.-The bulb of the thermometer was clyindrical in shape, 1.25 inches in length by 0.25 inch in diameter. The thermometer had an over-all length of 12 inches, and was very similar to T-11. Lag, 26 seconds. 
B. S Journal of Research, RP222

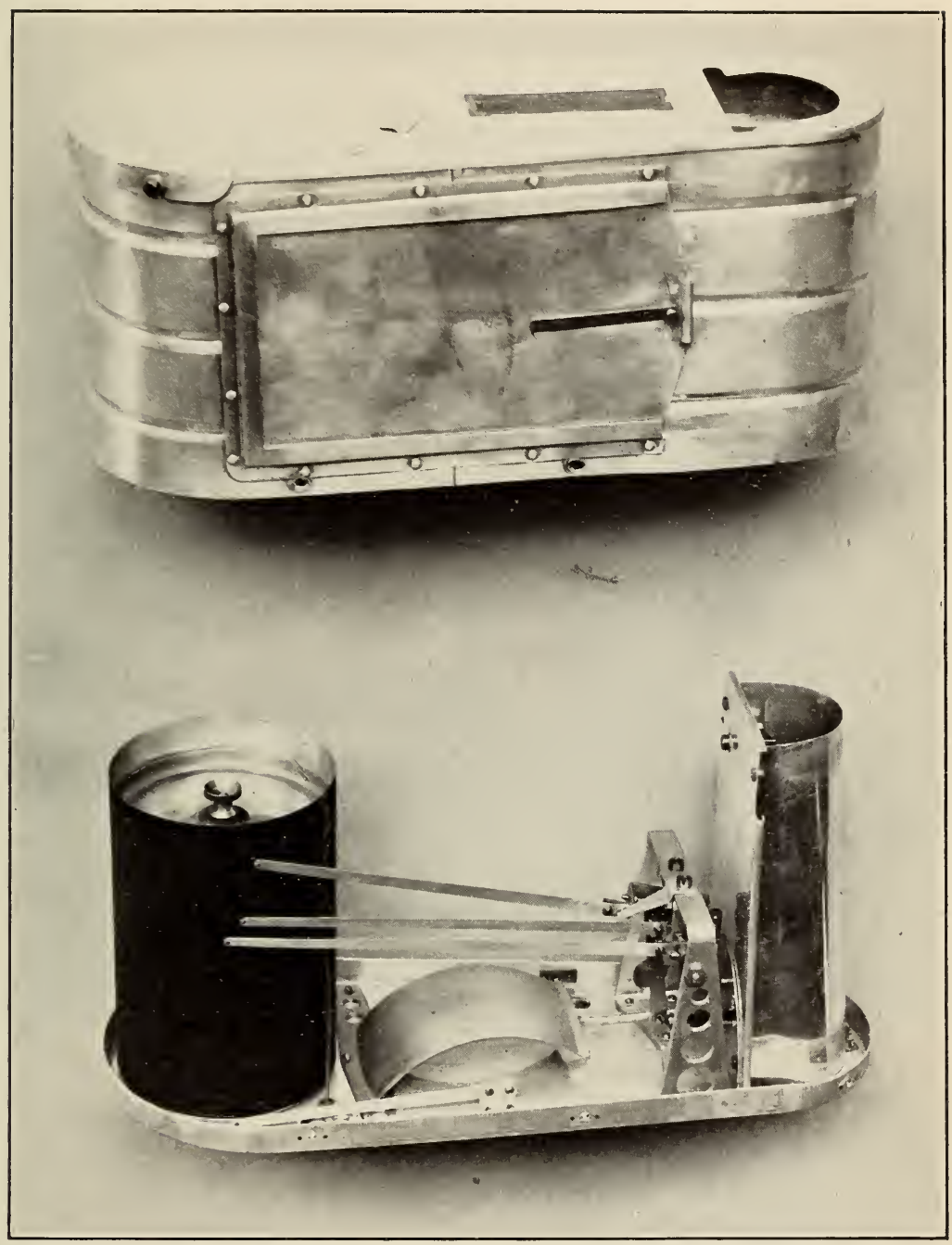

FIGURE 9.-Fergusson meteorograph

The bimetallic temperature element is mounted in the channel at the right end of the instrument. 
B. S. Journal of Research, RP222

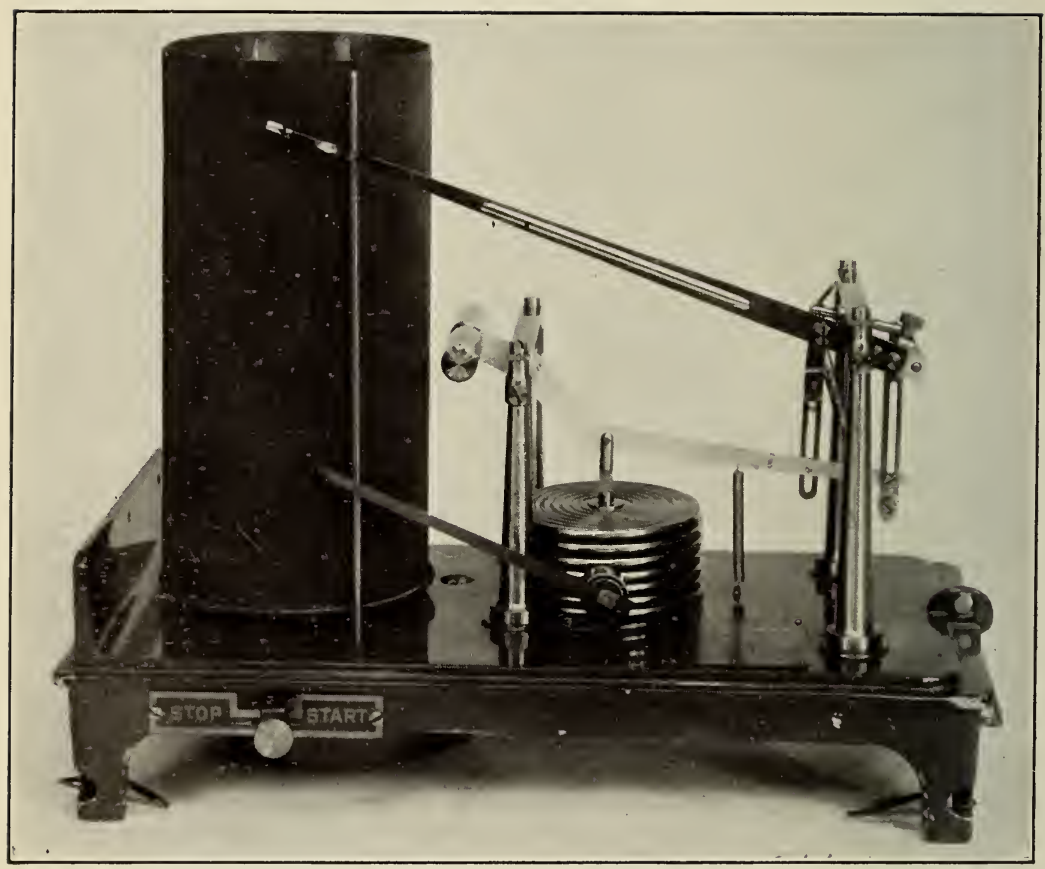

Figure 10.-Dual traverse barograph with cover removed

The lower stylus records the instrument temperature. 
T-13. Laboratory thermometer, mercury-in-glass type.-The bulb was cylindrical in shape, 0.5 inch in length by 0.19 inch in diameter. The instrument had an over-all length of 10 inches. As the thermometer was filled with mercury, the lag was somewhat less than for a similar instrument filled with a liquid such as pentane. The bulb of the instrument is shown in sketch T-13 of Figure 3. Lag, 18 seconds.

T-14. Laboratory thermometer, mercury-in-glass type.-The bulb of this thermometer was very similar to $\mathrm{T}-13$. It was cylindrical in shape, 0.63 inch in length by 0.19 inch in diameter. The instrument had an over-all length of 9 inches. Lag, 17 seconds.

T-15. Electric resistance thermometer, model No. 1.-This instrument was constructed for the National Advisory Committee for Aeronautics by the Bureau of Standards. T-15 was the first of a series of experimental types of resistance elements which were constructed. It is shown in the sketch T-15 of Figure 7. The element consisted of a three-layer coil of No. 36 single silk-covered nickel wire, wound on a thin, hollow, metallic bobbin 2.25 inches long by 0.62 inch in diameter. The resistance was $205 \mathrm{ohms}$ at $+20^{\circ} \mathrm{C}$. Holes were bored into the outside protecting tubing so as to reduce the thermal lag. For this particular element the lag was found to be 17 seconds (the average of six determinations) in an air stream of 17 miles per hour. The lag was found to be 22 seconds (the average of two determinations) in an air stream of 10 miles per hour.

T-16. Thermograph, flat bimetallic strip temperature element.This instrument was designed and built by the Bureau of Standards for the Bureau of Aeronautics, Navy Department, for the purpose of recording free-air temperatures in flight test work. The invar-brass bimetal temperature element was very similar to that shown in the sketch $\mathrm{T}-17$ of Figure 7 . It measured 2.75 inches long, 1 inch wide at the fixed base of the strip tapering to 0.75 inch at the end, and 0.030 inch thick. The strip was insulated from the aluminum base of the thermograph by mounting it upon a block of bakelite. Lag, 15 seconds.

T-17. Thermograph, bimetallic strip temperature element.-The thermal element of this recording instrument of foreign make was very similar to T-16 described above. The flat strip, 2.5 inches long by 1 inch wide by 0.023 inch thick, is shown in the sketch T-17 of Figure 7. Lag, 13 seconds.

T-18. Electric resistance thermometer, model No. 2.-This element was designed and constructed for the National Advisory Committee for Aeronautics. The temperature element consisted of No. 40 single silk covered nickel wire, wound around a rectangular piece of brass, 0.093 inch thick by 2.56 inches long by 0.87 inch wide, of which the inner portion was removed so as to leave an eighth of an inch margin all around. The nickel wire, $200 \mathrm{ohms}$ at $26^{\circ} \mathrm{C}$., was secured to the brass frame with bakelite varnish. This frame was then mounted within a perforated housing of 0.09 inch brass, 1.12 inches wide by 2.56 inches long, in order to give it mechanical support and protection from the sun. The over-all length including the projecting ends of the base of the box was 7.75 inches. The projecting ends afford a ready means for attaching the element to the airplane. The sides of the protective casing were perforated with many holes in order to secure adequate ventilation of the inclosed temperature element. 
The element in its housing is shown in sketch T-18 in Figure 7. Lag, 12 seconds.

T-19. Thermograph of Fergusson meteorograph.-This meteorograph was of the type used by the Weather Bureau in sounding balloons for the purpose of obtaining temperature, pressure, and humidity data of the upper atmosphere. It is shown in Figure 9. It was made almost entirely of aluminum sheeting about 0.008 inch in thickness. The weight of the entire instrument was 7 ounces and the over-all dimensions, 8.25 inches in length, 3.5 inches in height, and 3.37 inches in the greatest width. The temperature element was of phosphor-bronze, invar-steel bimetal and was made in the form of a three-fourths closed collar, 1 inch wide, 0.62 inch in diameter, and 0.012 inch thick. The element was mounted in a channel open at both ends with the axes of the element and the channel concentric. The fixed end of the element was attached to an insulating block of bakelite, 1 inch long by 0.31 inch square.

When testing the instrument in the wind tunnel, the axis of the air channel was at an angle of approximately $45^{\circ}$ with respect to that of the tunnel. When testing for lag in still air, the air channel was in the vertical position and both ends were kept uncovered and free from obstruction. The time lag was 11 and 20 seconds, when the air stream in the wind tunnel was 17 and 10 miles per hour, respectively. In still air the lag was three minutes.

T-20. Electric resistance thermometer, model No. 3.-This design of the temperature element for the electric resistance thermometer (with minor modifications) was found to be the most satisfactory, and is the type with which the instruments constructed by the Bureau of Standards for the National Advisory Committee for Aeronautics and Bureau of Aeronautics of the Navy Department were finally equipped. In this design a decided reduction in lag was obtained. A No. 40 single silk covered nickel wire was wound in a single layer around a thin copper cylinder that was equipped with internal, length-wise, radiation fins. This copper shell was only 0.002 inch in thickness. In addition to their function of radiating heat, the internal fins also served to strengthen the cylinder. The cylinder measured 0.50 inch in diameter by 2 inches in length. The nickel wire, $200 \mathrm{ohms}$ at $-20^{\circ} \mathrm{C}$., was fastened to the cylinder with bakelite varnish which has a comparatively high thermal conductivity. The temperature element was protected from the direct rays of the sun as well as from mechanical injury by inserting it lengthwise and concentric within a piece of fairly heavy-walled brass tubing which measured 0.75 inch in diameter and 2.25 inches in length. The temperature element unit was supported within the protective tubing by means of three thin metal strips spaced $120^{\circ}$ apart at each end of the tube. This construction is illustrated in sketch T-20 of Figure 7. A photograph of the entire instrument with slightly modified design of temperature element, but of substantially the same time lag as T-20 is shown in Figure 8. Lag, four seconds.

T-22. Resistance temperature element.-An experimental temperature element consisting of nickel wire woven over seven parallel cotton strands was mounted on a light brass frame so as to be easily handled during tests. This is shown in T-22 of Figure 7. The wire was No. 40 single silk covered nickel, $200 \mathrm{ohms}$ at $+20^{\circ} \mathrm{C}$., woven into a rectangular mat 2.75 inches long by 0.62 inch wide. This mat 
was mounted in the wind tunnel with its length parallel to that of the tunnel. Tests on the element made with and without a coating of bakelite varnish resulted in the same lag. Tests were made in air streams of 10 and 17 miles per hour speeds giving lags of four and three seconds, respectively.

\section{RESULTS OF TESTS ON BAROGRAPHS}

The results of the time lag determinations on five typical barographs, made in a manner somewhat similar to the tests on the thermometers and thermographs, are given in the following paragraphs. In each case the tests were made with the instrument covers in place, which is the service condition. The lag was determined first in still air, and then in air moving with a speed of 10 miles per hour. Barographs in service receive little or no ventilation. Tests in the 10 miles per hour air stream were made in order to ascertain the effects of a moderate amount of ventilation. A coiled bimetal temperature indicator, similar to T-10 described above, was installed successively on each barograph tested. This coil was securely fastened to the metal base-plate, as close as possible to the pressure element. The lag of thermometer bulbs is usually expressed in seconds, whereas the lag of barographs is more conveniently expressed in minutes. A plot, similar to that in Figure 1 which shows the time lag results for thermometers, is given in Figure 2 for the barographs.

$B-1$. Common barograph, wooden case.-This barograph had a mahogany casing 0.31 inch thick, covering a wooden base which was somewhat thicker. The recording drum and pressure capsules were mounted on a heavy brass base 0.09 inch thick, by 3.75 inches long, by 3 inches wide. The over-all dimensions of the wooden casing were 7 inches in length, by 5 inches in height, by 4.37 inches in width. A glass cover, 5.75 inches long by 3 inches wide, occupied most of the front side of the case. The total weight was 3 pounds and 4 ounces. Lag in still air, 74 minutes. Lag in an air stream of 10 miles per hour, 14 minutes.

B-2. Common barograph, aluminum case.-This barograph had an aluminum casing 0.31 inch thick. The recording drum and pressure capsules were mounted on an aluminum base plate 0.08 inch thick, by 4.75 inches long, by 3 inches wide. This plate was mounted upon four small bushings so that there was a quarter of an inch clearance between it and the bottom of the case. The over-all dimensions of the aluminum casing were 6.5 inches in length, by 4.5 inches in height, by 4 inches in width. A full-sized celluloid window was fitted into the front side of the instrument. The weight of the barograph was 1 pound and 5 ounces. Lag in still air, 25 minutes. Lag in an air stream of 10 miles per hour, 9.5 minutes.

$B$-3. Dual-traverse barograph.-This dual-traverse barograph was of the type commonly used in high altitude flights and in flight test work. The instrument with the cover removed is shown in Figure 10. This view also shows the coiled bimetal temperature indicator used in making the lag tests. The barograph was made entirely of metal, with a heavy aluminum base and a painted sheet copper casing. The over-all dimensions were 8.5 inches in length, by 7.5 inches in height, by 4.5 inches in width. The total weight was 5 
pounds and 2 ounces. Lag in still air, 22 minutes. Lag in an air stream of 10 miles per hour, 5 minutes.

B-4. Single-traverse barograph.-This barograph was of the same make and general case construction as the above instrument. The size and weight, however, were just about one-half those of the dualtraverse instrument. The over-all dimensions were 8 inches in length, by 5 inches in height, by 3.75 inches in width. The weight was about 3 pounds. Lag in still air, 22 minutes. Lag in an air stream of 10 miles per hour, 5 minutes.

\section{SUMMARY}

\section{THERMOMETERS, THERMOGRAPHS, AND METEOROGRAPH}

The time lag of 22 temperature elements of thermometers and thermographs when ventilated by an air stream of 17 miles per hour has been determined. The results are given in the following list which is arranged in the order of decreasing values of the lag:

Instrument

Time $\operatorname{lag} \lambda$ (in seconds)

T-1. Thermograph, liquid-in-metal tubes _... 72

T-2. Strut thermometer, liquid-in-glass bulb 57

T-3. Do...

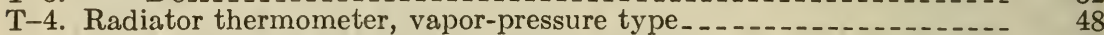

T-5. Thermograph, Bourdon tube, liquid-filled ..... 46

T-6. Strut thermometer, distant reading, liquid-filled.... 39

T-7. Thermograph, Bourdon tube, liquid-filled ...... 33

T-8. Strut thermometer, liquid-in-glass _... 32

T-9. Strut thermometer, liquid-in-metal helical tube... 30

T-10. Minimum-temperature-recording strut thermometer...... 29

T-11. Laboratory thermometer, liquid-in-glass .......... 28

T-12. Do

T-13. Laboratory thermometer, mercury-in-glass

T-14. Do...

T-15. Electric resistance thermometer, model No. 1....... 17

T-16. Thermograph, flat bimetallic strip temperature element...... 15

T-17. Thermograph, bimetallic strip temperature element...... 13

T-18. Electric resistance thermometer, model No. 2 ...

$\mathrm{T}-19$. Thermograph of Fergusson meteorograph 11

T-20. Electric resistance thermometer, model No. 3

T-22. Wire-weave electric resistance temperature element......... 3

In still air the lag of thermograph T-19 was three minutes.

\section{BAROGRAPHS}

The time lag of four typical aircraft barographs has been determined in still air as well as in an air stream of 10 miles per hour. The results, again listed in the order of decreasing lag values, are given in the following table. 


\begin{tabular}{|c|c|c|}
\hline \multirow[b]{2}{*}{ Instrument } & \multicolumn{2}{|c|}{ Time lag- } \\
\hline & $\begin{array}{l}\text { In still } \\
\text { air }\end{array}$ & $\begin{array}{l}\text { In air at } \\
10 \\
\text { m. p. h. }\end{array}$ \\
\hline $\begin{array}{l}\text { B-1. Common barograph, wooden case } \\
\text { B-2. Common barograph, aluminum case } \\
\text { B-3. Dual-traverse barograph } \\
\text { B-4. Single-traverse barograph }\end{array}$ & $\begin{array}{r}\text { Minutes } \\
74 \\
25 \\
22 \\
22\end{array}$ & $\begin{array}{c}\text { Minutes } \\
14 \\
9.5 \\
5.0 \\
5.0\end{array}$ \\
\hline
\end{tabular}

In general, the metal cased barographs have considerably less thermal lag than the wooden cased instruments. Moderate ventilation, such as in an air stream of 10 miles per hour, reduces the lag by approximately one-fourth.

Washington, June 7, 1930. 